\title{
Higgs Boson Mass and Other Constants Estimated by Polymer Statistics Methods
}

\author{
Andrei Yakunin \\ Laboratory of Polymer Materials, Karpov Institute of Physical Chemistry, Moscow, 105064, Russia \\ *Corresponding Author: andreiyakunin@yandex.ru
}

Copyright (C) 2014 Horizon Research Publishing All rights reserved.

\begin{abstract}
The Higgs boson mass, the fine structure constant, the Weinberg angle, the critical exponents are estimated by polymer statistics methods. Friction forces have electroweak nature. New phase transitions by changing the even number of components of an ordering field, $n$, near the $\alpha$ - relaxation transition point in melt-crystallized polymers is revealed. The transition from $n=0$ to $n=2$ is found. In frameworks of scaling theory of phase transitions and critical phenomena the results obtained are in a good agreement with experimental and theoretical data.
\end{abstract}

Keywords Critical Exponent, Phase Transition, Higgs Boson Mass

\section{Introduction}

About the $\alpha$-relaxation transition the draw ratio at break, $\lambda_{\mathrm{br}}$, has a maximum $[1,2]$ in copolymers of low pressure ethylene - acrylic acids with the small number of the latter comonomer $(\sim 0.1-0.3 \mathrm{~mol} \%[1,3])$ as well as in linear polyethylene (PE) (see [4] and refs. in it). At this point the expression $\lambda_{\mathrm{n}}{ }^{2} / \lambda_{\mathrm{br}}{ }^{2}=P(N)$ (obtained for room temperature) should be changed as formulated in [2]. Here, $\lambda_{\mathrm{n}}$ is the neck draw ratio, $P(N)=l_{\mathrm{a}} / l_{\mathrm{a}}{ }^{\text {cr }}$ is the probability of collision of chain ends, $l_{\mathrm{a}}$ is the mean thickness of amorphous layers in isotropic material, $l_{\mathrm{a}}{ }_{\mathrm{ar}}^{\mathrm{cr}}$ is the same value at $N=N_{\mathrm{cr}}, N=M_{\mathrm{w}} / \mu$ is the polymerization degree, $\mu=14 \mathrm{~kg} / \mathrm{kmol}$ is the molecular mass of repeated $\mathrm{CH}_{2}$ group, $\ln N_{\mathrm{cr}} \approx 15.89$. Figure 1 shows the dependence of $\lambda_{\mathrm{br}}$ on $N$ at room [5] and elevated [4] temperatures. The theoretical curves $\lambda_{\mathrm{br}}=\left(N / N_{\mathrm{cr}}\right)^{(1-v d) / 2}$ are also presented in Figure 1, $v$ is the critical exponent of correlation radius, $d$ is the space dimension. It has been recently shown [6] that $v=0.5(1+t-1 / t)$ where $t=1+2 \pi(n$ $+2) / f, f=137, n$ is the number of components of ordering field; it should be underlined that $n$ may be only by the even number, otherwise, the gauge symmetry breaks (see Appendix). The theoretical curves in Figure 1 seem to confirm our assumption that near the $\alpha$-relaxation transition the phase transition from $n=0$ to $n=2$ occurs. Here, we have used precise value for $\ln N_{\mathrm{cr}} \approx 15.69$ in comparison with [2].

\section{Results and Discussion}

\subsection{Two Phase Model of Polymer Structure; An Estimation of Density Ratio}

Let a polymer chain with the polymerization degree, $N$, walk over two lattices having a total boundary, both being the simple cubic. In that case $z$ is the number of neighbour monomers surrounding a site of the crystalline lattice $(z=2 d$ $=6$ for three-dimensional space). If we will regard $z^{*}$ as the same parameter for the second amorphous lattice and introduce the probability $p=z * / z<1$ then we can write the partition function, $Z$ :

$$
Z=\sum_{N=0}^{\infty} p^{N}=1 /(1-p)
$$

where $p$ can be defined as the probability discovering a monomer of the chain in the first phase while $1-p$ is the same probability for the second phase. Assuming $Z=z^{*}$ we find

$$
1=Z(1-p)=p z(1-p)
$$

From the solution of the equation (1) we obtain

and

$$
\begin{gathered}
p_{ \pm}=0.5\left[1 \pm(1-4 / z)^{1 / 2}\right] \\
Z=3+3^{1 / 2} \approx 4.732: \\
Z / z \equiv p=p_{+}=1-p_{-}=1-1 / Z .
\end{gathered}
$$

The other solution $p_{-}=1 / Z=1-p$ has no interest for subsequent considerations. Taking the logarithm of the expression (1) we obtain

$$
\ln Z=\ln p+\ln z
$$

Multiplying the equation (2) by $c / \ln z$ where $c$ is the mean concentration of chain monomers in the first phase we find

$$
c \ln Z / \ln z=c \ln p / \ln z+c=C
$$

where $C$ is a mean concentration. We suppose that $C$ is the concentration of monomers of the chain in the second phase 
(Table 1).

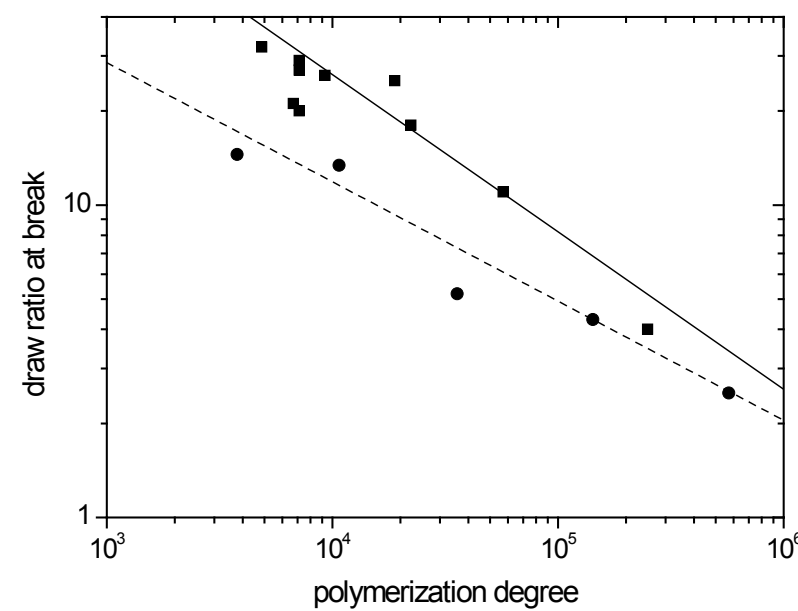

Figure 1. The draw ratio at break, $\lambda_{\mathrm{br}}$, vs the polymerization degree, $N$. Experimental data are obtained at room temperature [5] and at 75C [4], they are marked by circles and by squares, respectively; the theoretical curves $\lambda_{\text {br }}=\exp \left(-0.5(v d-1) \ln \left(N / N_{\text {cr }}\right)\right)$ correspond to $N_{\text {cr }} \approx \exp (15.69)$ and $v$ from Table 2 for $n=0$ (dash line) and $n=2$ (solid line)

Table 1. The density ratio of the crystalline phase to the amorphous one for some polymers. The theoretical value is 1.153 .

\begin{tabular}{|c|c|}
\hline polymer & $\begin{array}{c}\text { the ratio } \\
\text { densities, } \rho_{d} / \rho_{a}\end{array}$ \\
\hline polyethylene & 1.177 \\
\hline polyester, $-\left(\mathrm{CH}_{2}\right)_{2}$-O-CO- $\left(\mathrm{CH}_{2}\right)_{\mathrm{k}^{-}}, \mathrm{k}=8$ & 1.167 \\
\hline trans-1-4-polybutadiene & 1.163 \\
\hline trans-1-4-poly-2-methylbutadiene, $\alpha-$ form & 1.161 \\
\hline polyester, $-\left(\mathrm{CH}_{2}\right)_{2}$-O-CO- $\left(\mathrm{CH}_{2}\right)_{\mathrm{k}}-, \mathrm{k}=6$ & 1.152 \\
\hline polytetrafluoraethylene & 1.151 \\
\hline polyoximethylene & 1.126 \\
\hline polymeric selenium & 1.125 \\
\hline cis-1-4-polybutadiene & 1.122 \\
\hline polyvinyledenefluoride & 1.122 \\
\hline polyester, $-\left(\mathrm{CH}_{2}\right)_{2}$-O-CO- $\left(\mathrm{CH}_{2}\right)_{\mathrm{k}}-, \mathrm{k}=4$ & 1.111 \\
\hline isotactic polypropylene & 1.110 \\
\hline
\end{tabular}

Thus, we can draw an important conclusion: for the two-phase model of polymer structure the density ratio of the most dense phase to the second phase is the constant which is equal to

\section{$\ln z / \ln Z \approx 1.153$}

for three-dimensional space. It should be underlined that this relation is correct within the error which is less than $4 \%$ for a number of semicrystalline polymers. In Table 1 the experimental data [7] are presented for 12 polymers. The list may be expanded to a great extent.

The value of $Z \approx 4.732$ is very close to that of 4.68 which is a characteristic of the polymer statistics for the excluded volume problem [8]. This enables us to solve our problems in frameworks of the scaling theory of critical phenomena. This is the second important conclusion obtained by help of the present model.

\subsubsection{D-exponents}

In Table 2 the theoretical values of critical exponents $v, \gamma, \beta$ are presented. If $n=1$ or $n=3$ then such systems can be regarded as examples of materials when states with $n=0$ and $n=2$ (or $n=2$ and $n=4$ ) must have the same probability, while $n=6$ seems to be realized in some percolation systems such as gels [8]. The experimental value of the exponent $\eta$ is not determined exactly. The theoretical estimations $[9,10]$ show its weak dependence on $n$. In this connection, we may attribute any reasonable meaning to $\eta$. Then for $\beta$ and $\gamma$ the following evaluations can be obtained by standard ways $(\gamma+\beta=\delta \beta)[9]$ if to suppose $\eta=\delta / \mathrm{f}$ and to use the identity $\delta=-1+2 d /(d-2+\eta)$. Thus, $\eta \approx 0.0350, \gamma=$ $v d(\delta-1) /(\delta+1), \beta=v d /(\delta+1)$. These values are presented in Table 2. They are in a good accordance with recent theoretical data [10]. However, more accurate experimental checking could be made in future. If $1 / \mathrm{f}=0$ and $d=4$ then we obtain the Landau exponents.

Thus, we can see that friction forces have electroweak nature. Firstly, $\lambda_{\mathrm{n}}$ is expressed by help of the $\beta$-exponent and connected with the energy dissipation during drawing $[2,3]$. Secondly, the exponent $\eta$ is dealing with a dynamic exponent $[2,3,8]$ and characterized the viscosity of swelling polymer coil $[2,3]$.

Table 2. Critical exponents to the 4 th decimal place.

\begin{tabular}{|c|c|c|c|c|}
\hline$n$ & $v$ & $\gamma$ & $\beta$ & $(v d-1) / 2$ \\
\hline 0 & 0.58787 & 1.15516 & 0.30423 & 0.38181 \\
\hline 2 & 0.66923 & 1.31503 & 0.34633 & 0.50385 \\
\hline 4 & 0.74549 & 1.46487 & 0.38579 & 0.61823 \\
\hline 6 & 0.81766 & 1.60669 & 0.42314 & 0.72649 \\
\hline 1 & 0.62927 & 1.23650 & 0.32565 & 0.44390 \\
\hline 3 & 0.70793 & 1.39106 & 0.36636 & 0.56189 \\
\hline
\end{tabular}

\section{Conclusion}

If the fine structure constant

$$
\alpha_{\mathrm{fs}}=e^{2} / \hbar c
$$

(where $e$ is the electron charge, 2 $\pi \hbar$ is the Planck constant, $c$ is the light velocity) will be approximately correspond to $\mathrm{f}^{-1}$ $=1 / 137$ then the universality of the critical laws should not be explained - everything is clear. In this case $\eta$ may be connected with the gauge constant $g=\eta^{-1 / 2}$ of a field, for example, $\sin \theta_{\mathrm{W}}=e / g \approx 0.457$ if $\theta_{\mathrm{W}}$ is the Weinberg angle in the theory of electroweak interactions. Supposing $N_{\text {cr }}=$ 
$M_{\mathrm{H}} / m_{\mathrm{e}}$ we find the Higgs boson mass $M_{\mathrm{H}}=\exp (15.69) m_{\mathrm{e}} \approx$ $3.33 \mathrm{TeV}$ where $m_{\mathrm{e}} \approx 0.511 \mathrm{MeV}$ is the electron mass.

The rest exponents are expressed in terms of the similarity laws [8-10]. Friction forces have electroweak nature.

\section{Appendix}

Following Edwards [11] a behaviour of polymer chain with the excluded volume can be described by help of function, $\psi(\boldsymbol{r})$, which is the solution of the diffusion equation

$$
\partial \psi / \partial N=\left[\left(a^{2} / 2 d\right) \nabla^{2}-\varphi(\boldsymbol{r}) / T\right] \psi=\hat{\mathrm{H}} \psi
$$

with the boundary condition $\psi\left(\boldsymbol{r}, \boldsymbol{r}^{`}\right)=\delta\left(\boldsymbol{r}-\boldsymbol{r}^{`}\right) a^{3}$ at $N=0$, $a$ is the monomer diameter (the lattice constant); $\varphi(r)$ is the potential connected with the excluded volume parameter, $V$, as follows

$$
V=\int[1-\exp (-\varphi(\boldsymbol{r}) / T)] \mathrm{d}^{3} \boldsymbol{r} .
$$

Let $\psi(\boldsymbol{r})$ be an eigen function of the operator $\hat{\mathrm{H}}(\boldsymbol{r})$. Let us assume also that there exists an uniform field $\boldsymbol{H}$ with a vector potential $\boldsymbol{A}(\boldsymbol{r})$. While the symmetry of the operator $\hat{\mathrm{H}}(\boldsymbol{r})$ can be broken due to the vector potential is not the periodic function of coordinates, a physical translation symmetry of the polymer system does not change, as the lattice over that the macromolecule makes random walks is periodic. Further we will use the well-known solution about the symmetry of electron states in the lattice with a magnetic field [12]. Let us choose the gauge for the vector potential of the uniform field:

$$
\boldsymbol{A}=\boldsymbol{H} \times \boldsymbol{r} / 2 .
$$

Upon a translation $\boldsymbol{r} \rightarrow \boldsymbol{r}+\boldsymbol{L}$ (where $\boldsymbol{L}$ is one of the lattice periods) $\psi(\boldsymbol{r}) \rightarrow \psi(\boldsymbol{r}+\boldsymbol{L})$. The latter is the eigen function of the $\hat{\mathrm{H}}(\boldsymbol{r}+\boldsymbol{L})$ which does not coincide with $\hat{\mathrm{H}}(\boldsymbol{r})$ due to substituting

$$
A(\boldsymbol{r}+\boldsymbol{L})=\boldsymbol{A}(\boldsymbol{r})+\boldsymbol{H} \times \boldsymbol{L} / 2
$$

for $\boldsymbol{A}(\boldsymbol{r})$. To return to the initial $\hat{\mathrm{H}}(\boldsymbol{r})$ the following gauge transformations are necessary

$$
\boldsymbol{A}(\boldsymbol{r}+\boldsymbol{L}) \rightarrow \boldsymbol{A}(\boldsymbol{r}+\boldsymbol{L})+\nabla f, f=-\boldsymbol{H} \times \boldsymbol{L} \cdot \boldsymbol{r} / 2 .
$$

The wave function $\psi(\boldsymbol{r}) \sim \exp (\mathrm{i} \boldsymbol{k} \cdot \boldsymbol{r})$ is the eigen function of the operator $\hat{\mathrm{H}}(\boldsymbol{r})$ (if $\varphi(\boldsymbol{r})=0$ in (A.1)) as it may be seen from the solution the equation

$$
\text { -i } \nabla \psi(\boldsymbol{r})=\boldsymbol{k} \psi(\boldsymbol{r}) .
$$

Under the gauge transformations the wave function must be multiplied by $\mathrm{e}^{\mathrm{i} z} f$ where $\mathrm{z}=$ const:

$$
\begin{gathered}
{[-\mathrm{i} \nabla-\mathrm{z} \boldsymbol{A}(\boldsymbol{r})] \psi \rightarrow[-\mathrm{i} \nabla-\mathrm{z} \boldsymbol{A}(\boldsymbol{r})-\mathrm{z} \nabla f] \mathrm{e}^{\mathrm{i} z f} \psi=-\mathrm{i} \nabla\left(\mathrm{e}^{\mathrm{i} z f} \psi\right)-} \\
\mathrm{e}^{\mathrm{i} z f} \psi[\mathrm{zA}(\boldsymbol{r})+\mathrm{z} \nabla f]=\mathrm{e}^{\mathrm{i} z f}[-\mathrm{i} \nabla-\mathrm{z} \boldsymbol{A}(\boldsymbol{r})] \psi .
\end{gathered}
$$

Let the translation operator $\hat{\mathrm{S}}_{L}$ be the result of all mentioned above actions:

$$
\hat{\mathrm{S}}_{L} \psi(\boldsymbol{r})=\psi(\boldsymbol{r}+\boldsymbol{L}) \exp (\mathrm{i} \boldsymbol{h} \times \boldsymbol{L} \cdot \boldsymbol{r} / 2)
$$

where $\boldsymbol{h}=\mathrm{z} \boldsymbol{H}$. One can obtain

$$
\hat{\mathrm{S}}_{L} \hat{\mathrm{S}}_{L}{ }^{\prime}=\hat{\mathrm{S}}_{L+L}{ }^{\prime} \mathrm{g}\left(\boldsymbol{L}, \boldsymbol{L}{ }^{\prime}\right)
$$

where

$$
\mathrm{g}\left(\boldsymbol{L}, \boldsymbol{L}^{\prime}\right)=\exp \left(-\mathrm{i} \boldsymbol{h} \cdot \boldsymbol{L} \times \boldsymbol{L}^{{ }^{\prime} / 2}\right)
$$

and

$$
\hat{\mathrm{S}}_{L} \hat{\mathrm{S}}_{L}=\hat{\mathrm{S}}_{L} \cdot \hat{\mathrm{S}}_{L} \mathrm{~g}^{2}\left(\boldsymbol{L}, \boldsymbol{L}{ }^{\prime}\right) .
$$

If the field $\boldsymbol{h}$ will be

$$
\boldsymbol{h}=(4 \pi / \mathrm{f}) v^{-1} \boldsymbol{a}_{3}
$$

where $\mathrm{f}$ is a simple number, $\boldsymbol{a}_{3}$ is one of the lattice periods, and $v$ is the volume of the elementary unit $\left(v=a^{3}\right.$ for the primitive cubic lattice which we will use for the sake of simplicity), then for the translations

$$
\boldsymbol{L}=a(m \mathbf{i}+\mathrm{f} n \mathbf{j}+l \mathbf{k})
$$

where $\mathbf{i}, \mathbf{j}, \mathbf{k}$ are the unit vectors of the orthogonal basis, here, $m, n$, and $l$ are the whole numbers, $\mathrm{g}\left(\boldsymbol{L}, \boldsymbol{L}^{\prime}\right) \equiv 1$ in (A.2) and $\hat{\mathrm{S}}_{L} \hat{\mathrm{S}}_{L} \cdot=\hat{\mathrm{S}}_{L} \cdot \hat{\mathrm{S}}_{L}$.

Returning to estimations of the critical exponents we have found [13] that the wave vector $q$

$$
q a \sim N^{0.5-v}
$$

gives the main contribution to the integral for receiving the pair correlation function of concentration fluctuation. The wave vector $q$ has a very important meaning for polymer statistics, since it is connected with the fluctuation interactions of the chain ends, and, consequently, with the field $\boldsymbol{h}$ corresponding to the chain ends $[14,8]$.

Let $h$ designate $\boldsymbol{h} \cdot \boldsymbol{k}$ in (A.3) and let us choose $N=\exp$ [1/(ha $)]$. Then $N<N_{\text {cr }}$ at $h>h_{\text {cr }}$ where $N_{\text {cr }}$ is the value of the polymerization degree at which the transition from a swelling coil to ideal $\left(h \rightarrow h_{\mathrm{cr}}>0\right)$ can be revealed [13]. One may find

$$
\ln \left(N / N_{\mathrm{cr}}\right) \approx \Delta h /\left(h_{\mathrm{cr}} a\right)^{2}
$$

where $\Delta h=h-h_{\mathrm{cr}}$.

Let us assume

$$
q / q_{\mathrm{cr}}-1=h / h_{\mathrm{cr}}-1 .
$$

Using (A.5) and expanding the exponential form of (A.4) yields

$$
\begin{gathered}
q / q_{\mathrm{cr}}-1=\exp \left[(0.5-v) \ln \left(N / N_{\mathrm{cr}}\right)\right]-1 \approx \\
(v-0.5) \Delta h /\left(h_{\mathrm{cr}} a\right)^{2}
\end{gathered}
$$

From (A.6), (A.7) and (A.3) we write

$$
v=0.5+4 \pi / \mathrm{f}=0.5+2 \pi(n+2) / \mathrm{f}=t-0.5
$$

Where $n$ is the number of components of an ordering field, $\mathrm{f}=137$ to obtain the best agreement with the estimation $v \approx 1$ $-6^{-1 / 2}$ at $n=0[3,13,15], t=1+2 \pi(n+2) / f$. We have used the expression $v-0.5 \sim n+2$ by the Wilson $\varepsilon$-expansion [9, 16]. Thus, $n$ may be the even number only, otherwise, $g(\boldsymbol{L}$, $\left.\boldsymbol{L}^{\prime}\right)$ can have the pure imaginary value in (A.2).

The formula (A.8) can be rewritten in other way supposing $2 \pi(n+2) / \mathrm{f}$ by a small parameter

$$
v=0.5(1+t-1 / t) .
$$


The expression (A.9) seems to agree with the results [9, 16-18] (see refs in these works).

\section{REFERENCES}

[1] A. N. Yakunin, A. N. Ozerin, N. I. Ivantscheva et al. A study of the orientational stretching of an ethylene-acrylic acid copolymer at various deformation temperatures, Polymer Science U.S.S.R., Vol.30, No.4, 854-859,1988.

[2] A. N. Yakunin. A Study of Critical Phenomena and Dissipative Nanostructures in Self-Organizing Polymer Systems, Polym. Sci. A,Vol.54, No.2, 155-164, 2012.

[3] A. N. Yakunin. A Globule in a Stretching Field. The Role of Partial Melting During Drawing of Crystalline Polymers, Int. J. Polym. Mater.,Vol.22, No.1-4, 57-65, 1993.

[4] G. Capaccio, A. G. Gibson, I. M. Ward. In Ultra-High Modulus Polymers, eds. A. Cifferi, I. M. Ward, Applied Science, London, 1977.

[5] R. Popli, L. Mandelkern. Influence of structural and morphological factors on the mechanical properties of the polyethylenes, J. Polym. Sci.: Part B: Polym. Phys., Vol.25, No.3, 441-483, 1987.

[6] A. N. Yakunin. Gauge fields in dissipation processes, arXiv: cond-mat/0308008.

[7] B. Wunderlich. Macromolecular Physics, vol. 3, Academic Press, New York and London, 1980.

[8] P.-G. de Gennes. Scaling Concepts in Polymer Physics,
Cornell University Press, New York, 1979.

[9] K. G. Wilson, J. Kogut. The renormalization group and the E-expansion, Phys. Rep. C, Vol.12, No.2, 75-199, 1974.

[10] J. Z. Zinn-Justin. Precise determination of critical exponents and equation of state by field theory methods, Phys. Rep., Vol.344, No.4-6, 159-178, 2001.

[11] S. F. Edwards. The statistical mechanics of polymers with excluded volume, Proc. Phys. Soc., Vol.85, No.4, 613-624, 1965.

[12] E.M.Lifshitz,L.P.Pitaevskii.Statistical physics. Part 2. Theory of the condensed state, 3rd ed., Pergamon, Oxford, 1980.

[13] A. N. Yakunin. Nematic ordering problem as the polymer problem of the excluded volume, Cent. Eur. J. Phys., Vol.1, No.2, 355-362, 2003.

[14] J. des Cloizeaux. Lagrangian theory for a self-avoiding random chain, Phys. Rev. A, Vol.10, No.5, 1665-1669, 1974.

[15] P.-G. de Gennes. Exponents for the Excluded Volume Problem as Derived by the Wilson Method, Phys. Lett. A, Vol.38, No.5, 339, 1972.

[16] A. Z. Patashinskii, V. L. Pokrovskii. Fluctuation Theory of Phase Transitions, ed. P. J. Shepherd, Pergamon, Oxford, 1979.

[17] S. Caracciolo, M. S. Causo, A. Pelissetto. High-precision determination of the critical exponent $\gamma$ for self-avoiding walks, Phys. Rev. E, Vol.57, No.2, R1215-R1218, 1998.

[18] G. Besold, H. Guo, M. J. Zuckermann. Off-Lattice Monte Carlo Simulation of the Discrete Edwards Model, J. Polym. Sci.: Part B: Polym. Phys., Vol.38, No.8, 1053-1068, 2000. 